\title{
Antibiotic Prophylaxis in Gynecology, Oncology and Surgical Procedures a Brief up-to Date
}

\section{Panagiotis Peitsidis*}

Department of Obstetrics and Gynecology, South end University Hospital, UK

Infectious complications in Gynecology Oncology surgical procedures are related with significant morbidity and potential mortality. Several infectious complications may be confronted in gynecology oncology practice and these encompass intra abdominal infections, infections of the vaginal cuff, endometritis, wound infections, subcutaneous cellulitis, perineal infections, port site infections in laparoscopic and endoscopic interventions, cystitis etc. The hospitalization of the patient is prolonged and the health care costs are increased hence causing alertness to health trust services. Since the initiation of the antibiotic era administration, an enormous amount of studies and trials have been conducted leading the clinician to uncertainties which regimen to apply, what dose to give, when to give and for how long?

The purpose of antibiotic prophylaxis in surgical procedures is not to sterilize tissues but to reduce the colonization pressure of microorganisms introduced at the time of operation to a level that the patient's immune system is able to overcome. Prophylaxis does not prevent infection caused by postoperative contamination [1].

Surgery in gynecology oncology includes radical extensive abdominal and pelvic procedures with risk of postoperative infection. An important aspect in gynecology oncology and in surgery in general is to reduce the rate of surgical site infections (SSIs) which occur for 40 $\%$ of all noso comial infections in surgical patients and occur in up to 20 $\%$ of patients undergoing abdominal surgery; there is a twofold increase in mortality in surgical patients with SSIs [2]. It is widely accepted that preoperative that factors which increase preoperative infection rate in women undergoing radical pelvic surgery are associated with elongation of duration of surgery, patient's performance status, anemia, nutritional status, history of post surgical infections, metabolic diseases, presence of malignancy, post irradiation and post chemotherapy status, obesity etc [3].

Several microorganisms have been implemented as causative agents are coli forms, staphylococci, streptococci, clostridia, bacteroides. Several recommendations for antibiotic prophylaxis have been reported; the majority of authors recommend the administration of cephalosporin's which have broad spectrum coverage and half-life, especially cefazolin combined with metronidazole in cases where bowel resection maybe applied [4]. Cefazolin can be considered as alternative regimen in patients with penicillin allergy, furthermore other agents can be given such as clindamycin, gentamycin, erythromycin etc [4].

Antibiotic prophylaxis regimens must also be safe and inexpensive, the agent must be administered in a way that ensures that serum and tissue levels are adequate before an incision is made and that therapeutic levels of the agent can be maintained in serum and tissue [5].

The current evidence suggests that if prophylactic antibiotics are to be given, they should be administered shortly before or at bacterial inoculation. This should be performed 15 to 60 minutes before skin incision; a single dose is considered adequate for operations lasting more than three hours in addition a second dose should be given when blood loss exceeds $1500 \mathrm{~mL}^{3}$.
When a surgical site infection occurs; how long do we have to follow-up? It has been reported by Mangram et al. that surveillance for surgical site infection monitoring requires follow-up for 30 days postoperatively, and the trend towards early discharge from hospital makes surveillance a challenge. It is fact that I that up to $84 \%$ of surgical site infections occur following discharge from hospital [5].

Antibiotic resistance occurs from incorrect and inappropriate use of regimens. In addition to antibiotic prophylaxis, all factors that affect infectious risk reduction in gynecology oncology must be reviewed. Sterile surgical measures must be ensured, combined with ongoing quality of sterilization technique, monitoring of air ventilation, and postoperative wound care are required. Consistent infection control surveillance and reporting of infectious complications track ability to minimize these morbidities and possibly to identify clusters of infection and the emergence of antibiotic resistant organisms. This will dictate changes to operative routines to respond to evolving microbial diversity that seems inevitable [6].

\section{References}

1. Mangram AJ, Horan TC, Pearson ML, Silver LC Jarvis WR (1999) Guideline for Prevention of Surgical Site Infection, 1999. Centers for Disease Control and Prevention (CDC) Hospital Infection Control Practices Advisory Committee. Am J Infect Control 27: 97-132.

2. Mu Y, Edwards JR, Horan TC, Berrios-Torres SI, Fridkin SK (2011) Improving risk-adjusted measures of surgical site infection for the national healthcare safety network. Infect Control Hosp Epidemiol 32: 970-986.

3. Dellinger EP, Gross PA, Barrett TL, Krause PJ, Martone WJ, et al. (1994) Quality standard for antimicrobial prophylaxis in surgical procedures. Infectious Diseases Society of America. Clin Infect Dis 18: 422-427.

4. Cormio G, Di Fazio F, Lorusso F, Di Gesù G, Cacciapuoti C, et al. (2002) Antimicrobial prophylaxis in laparotomic gynecologic surgery: a prospective randomized study comparing amoxicillin-clavulanic acid with cefazolin. J Chemother 14: 618-622.

5. Mangram AJ, Horan TC, Pearson ML, Silver LC, Jarvis WR (1999) Guideline for Prevention of Surgical Site Infection, 1999. Centers for Disease Control and Prevention (CDC) Hospital Infection Control Practices Advisory Committee. Am J Infect Control 27: 97-132.

6. Van Eyk N, van Schalkwyk J (2012) Infectious Diseases Committee. Antibiotic prophylaxis in gynaecologic procedures. J Obstet Gynaecol Can. 34: 382-91.

*Corresponding author: Panagiotis Peitsidis, Department of Obstetrics and Gynecology, South end University Hospital, United Kingdom, E-mail: panagiotis_pp@yahoo.com.

Received March 25, 2013; Accepted March 27, 2013; Published March 31, 2013

Citation: Peitsidis P (2013) Antibiotic Prophylaxis in Gynecology, Oncology and Surgical Procedures a Brief up-to Date. Clin Microbial 2: e110. doi:10.4172/23275073.1000e110

Copyright: (c) 2013 Peitsidis P. This is an open-access article distributed under the terms of the Creative Commons Attribution License, which permits unrestricted use, distribution, and reproduction in any medium, provided the original author and source are credited. 